\title{
BENCHMARKING OF CELL THROUGHPUT USING PROPORTIONAL FAIR SCHEDULER IN A SINGLE CELL ENVIRONMENT
}

\author{
${ }^{1}$ Ramprasad Subramanian, ${ }^{1}$ Roshanak Heidari, ${ }^{1}$ Kumbesan Sandrasegaran, ${ }^{2}$ AMA \\ Dhanraj and ${ }^{2}$ Karthik S \\ ${ }^{1}$ School of Computing and Communications, Faculty of Engineering and Information \\ Technology, Centre for Real Time Information Networks, University of Technology \\ Sydney, Sydney, Australia. \\ ${ }^{2}$ Freelance Telecom Engineers
}

\begin{abstract}
The proportional fair (PF) scheduling algorithm compromises between cell throughput and fairness. Many research findings have been published by various researchers about PF algorithm based on mathematical model and simulations. In this paper we have taken the practical route to analyse the algorithm based on three types of subscription. In this benchmarking study, the user subscriptions are differentiated as Gold, Silver and Bronze schemes and they are provisioned with certain throughputs. Apart from subscriptions plans, the channel condition also plays a major role in determining the throughput. So in order to ensure fairness among different subscriptions even in the bad channel conditions and to deliver the provisioned throughputs certain priorities are attached with the subscriptions. As per the subscription plans Gold subscribers are assigned with 50\% of the speed offered by the network as maximum based on CAT3 speed (100 Mbps in DL and 50 Mbps in UL), Silver is assigned with 25\% of the max speed and Bronze is assigned with $12 \%$ of the max speed. The priorities assigned to subscribers determines the fairness in the unfavourable channel conditions - Bronze (high), Silver and Gold (medium). In this paper, an benchmarking tests have been performed with all of three types of subscribers for nearly two hours in the live single cell network without any heterogeneous cells influencing it. Furthermore, the results are compared with the simulation results.
\end{abstract}

\section{Keywords}

LTE; Proportional Fair (PF); Scheduling; Benchmarking; Single Cell; Throughput Analysis

\section{Introduction}

LTE system is evolved from the earlier 3GPP system known as Universal Mobile Telecommunication System (UMTS), which in turn was evolved from Global System for Mobile Communication (GSM). In 2004, 3GPP started the work on LTE. The main aim was to deliver high data rates with low latency. In the new architecture, the Circuit Switched (CS) core network was replaced by Packet Switched (PS) core network to takes care of voice and data traffic unlike its predecessor - UMTS, wherein the voice traffic is handled by CS core and data traffic is handled by PS core. Originally, the conceived theoretical value that was planned to achieve in 
International Journal of Wireless \& Mobile Networks (IJWMN) Vol. 7, No. 2, April 2015

LTE in peak with the data rate of $100 \mathrm{Mbps}$ in the downlink and $50 \mathrm{Mbps}$ in the uplink. But when the LTE network was introduced the peak data achieved was $300 \mathrm{Mbps}$ in downlink and $75 \mathrm{Mbps}$ in the uplink. In LTE, the communication is available in different frequency bands, of different sizes. Furthermore, the LTE uses both paired and un-paired bands for the communication. In the paired band, the same frequency is used in both the uplink and downlink, whereas in the unpaired band, the uplink and downlink communications uses different frequency bands. In LTE downlink transmissions, frame length of $10 \mathrm{~ms}$ is used and grouped by radio transmission. That is, each radio frame is created from 10 subframes of $1 \mathrm{~ms}$ duration. Therefore both the uplink and downlink uses 10 subframes. Thereafter, the subframes are divided into two slots with the duration of $0.5 \mathrm{~ms}$ for each part.

Traditionally telecommunication networks provides voice and data services with voice being the dominant but in recent times this usage pattern has been changed, the data usage has overtaken the voice. The growth in ubiquitous data service is fuelled by the rapid growth in internet and new services such as LTE/LTE-A are designed to support various high data rate services. To accommodate these requirements, the $3 \mathrm{GPP}$ cellular systems has evolved from circuit switches to IP enabled packet switches. The present day network architecture like LTE/LTE-A has evolved to a flat IP architecture to support the growing demand of the data hungry applications which is aptly supported by smart phones. So to provide high speed data services and better throughput, packet schedulers plays a major role. In the evolved architecture like LTE, packet scheduler plays an important role in the resource management. Several researches have proved that scheduling policy plays a very vital role that affects the system performance parameters such as throughput, delay, fairness and loss rate in wire line and wireless domains [2]. The major difference between the wire line and wireless environment is the time varying and location dependent feature of channel fading. Channel fading is one of the major phenomenon that needs to be considered when analysing issues related with throughput, delay, fairness and loss rate. But these are non-existent issue in wire line environment.

Most of the research studies in wireless packet scheduling use two states of the channel (the channel between the base station (BS) and the UE) conditions - the channel condition is assumed to be good or bad [3][4]. The packet transmission is always successful in good channel condition but whereas in bad channel condition the packet transmission always fails because of highly erroneous in nature. The performance of the schedulers is always compared with the reference model that describes the service status for each session in an error free environment. Some session falls short to the reference model and other might overwhelm the reference model. However, the discussed two state of the channel model for the schedulers are the weak points in the schedulers study. Since, the two states prescribe only two channel conditions, but reality the channel condition vary rapidly and to know the conditions BS requires the fast feedback from the UE on the channel condition but this comes with the prise of increasing signalling traffic. The IS856 standard (also known as high data rate (HDR) of QUALCOMM) proposes as an architecture that proposes to increase the better throughput with the aid of increasing the signalling overhead [5]. Several researchers have proposed several algorithms based on this [6][7][8][9][10]. Hereafter the terms UE and mobile terminals will be used interchangeably.

The schedulers that exploit the time varying channel conditions are considered in this paper. In an ideal condition the maximum throughout can be achieved but the fairness is compromised. Hence, a trade-off should be worked out between the throughput and fairness. One of the regularly used fairness scheme is max-min fairness, which maximises the minimum rate of the 
International Journal of Wireless \& Mobile Networks (IJWMN) Vol. 7, No. 2, April 2015

session based on the link capacity constraint. The fairness of this algorithm is questioned and therefore the PF scheduler was introduced in [1] which provides better fairness. In [11], the mathematically model for the PF scheduler algorithm is introduced. In this paper, field testing and benchmarking of PF scheduler algorithm is made. The test was conducted with varying subscription. The subscription ranges from Gold, Silver and Brown. Gold subscription is designed to receive $50 \%$ of the speed offered by the network as a max based on CAT3 speed (100 Mbps in DL and 50 Mbps in UL), Silver follows the next with $25 \%$ of the max speed and while the Bronze with least will get around $12 \%$ of the max speed.

\section{System model}

\section{A. System and Channel}

In the system model, it is considered that the downlink channel of a single cell wireless access network is applied, where $\mathrm{N}$ mobile terminals are serviced by $\mathrm{N}$ terminals. The downlink of PF is very much similar to IS-856 system where a single broadband channel is shared by all the mobile terminals present in the cell. The BS uses the pilot signal at the specified time slot is received by all the UEs present in the cell and the feedback on the channel condition and transmission rate is constantly fed to the system. Based on the received information from UE, the BS decides to serve the next UE. As per the scheduler, the BS transmits packets with the full power. As specified in [12], the transmitting power at the BS can be specified as $P_{t}$, the power received at the mobile is given by $P_{k}=\left|h_{k}\right|^{2} P_{t}$, where $h_{k}$ is the channel gain. The combination of various factors such as scattering, absorption, shadowing and multipath effect contributes to the channel gain. The channel gain between $\mathrm{BS}$ and the user $k$ can be written as:

$$
h_{k}=\sqrt{c d_{k}^{-\alpha}} S_{k} m_{k}
$$

In equation (1), $c$ is constant and it in-corporates the transmitting and receiving antenna gains, $d_{k}$ denotes the physical spacing between the BS and user $k, \alpha$ denotes path loss ( this is considered to be around 4.0 in the urban environment [11]), $S_{k}$ represents the shadowing effect, $m_{k}$ is the sum of the multipath components. The shadowing effect follows the log-normal distribution with zero-mean and variance $\sigma_{s}^{2}$ which is represented in decibels in the log scale. The multipath fading is modelled in second order chi-square or exponential random variable with mean 1.0, which represents the Rayleigh fading channel. Since we have considered the scenario of single cell, the intercell interference is not considered. Therefore, the SNR of user $k$ can be

represented as $Z_{k}=P_{k} / P_{n}$, where $P_{n}$ is the background noise power that includes thermal noise and other Gaussian interferences. In [11], the median SNR at the cell edge $\rho$ represents the noise level of the wireless environment that is defined. $\rho=c D^{-\alpha} P_{t} / P_{n}$, and in this $D$ can be specified as the radius of the cell, the average SNR of the user $k$ as $\overline{Z_{k}}=\rho\left(\frac{D}{d_{k}}\right) \alpha S_{k}$. The signal level follows the exponential distribution, and the noise power is constant. The SNR is also modelled by exponential random variable.

\section{B. PF Scheduler}

Many studies in the area of PF in the past have considered two states of channel model with the assumption that the transmission rate is fixed regardless of the channel state. With the bandwidth 
International Journal of Wireless \& Mobile Networks (IJWMN) Vol. 7, No. 2, April 2015

scarcity, cell throughput forms an important indicator to evaluate the wireless channel and this forms an important metric to evaluate the scheduling algorithms. With the given channel gain for each user, the base station (BS) serves the user $k$ first, whose magnitude of the channel gain is larger than other users. The PF schedulers is designed in such a way that it allocates a considerable portion of time slots to each subscribers while giving preference to the subscribers in good channel state. In the studied case, the subscribers are broadly classified as Gold, Silver and Bronze. Based on the subscription and tariff, the subscribers are classified as above. The exponential moving average tracks the average throughput of each user. The channel state is sent as the feedback by each user at the beginning of the every time slot. The feasible rate to the average throughput is calculated for each user by BS which forms a key selection criterion, in turn, it forms the basis of the preference metric and based on this preference metric, the transmission for forthcoming timeslots will be decided. Figure 1 illustrates the Fairness between the mobiles based on the channel condition. This can defined as the following. The possible user rate for the user $k$ in the time slot $n$ is $R_{k}[n]$, the moving average can be denoted as $\widetilde{R_{k}}[n]$. Then, user $k^{*}=\arg \max _{k} R_{k}[n] / \widetilde{R_{k}}[n]$ is served in $n$ time slots, and throughput for each user can be updated as below

$$
\widetilde{R_{k}}[n+1]=\left\{\begin{array}{c}
\left(1-\frac{1}{t_{c}}\right) \widetilde{R_{k}}[n]+\frac{1}{t_{c} R_{k}}[n], \quad k=k^{*} \\
\left(1-\frac{1}{t_{c}}\right) \widetilde{R_{k}}[n], \quad k \neq k^{*}
\end{array}\right.
$$

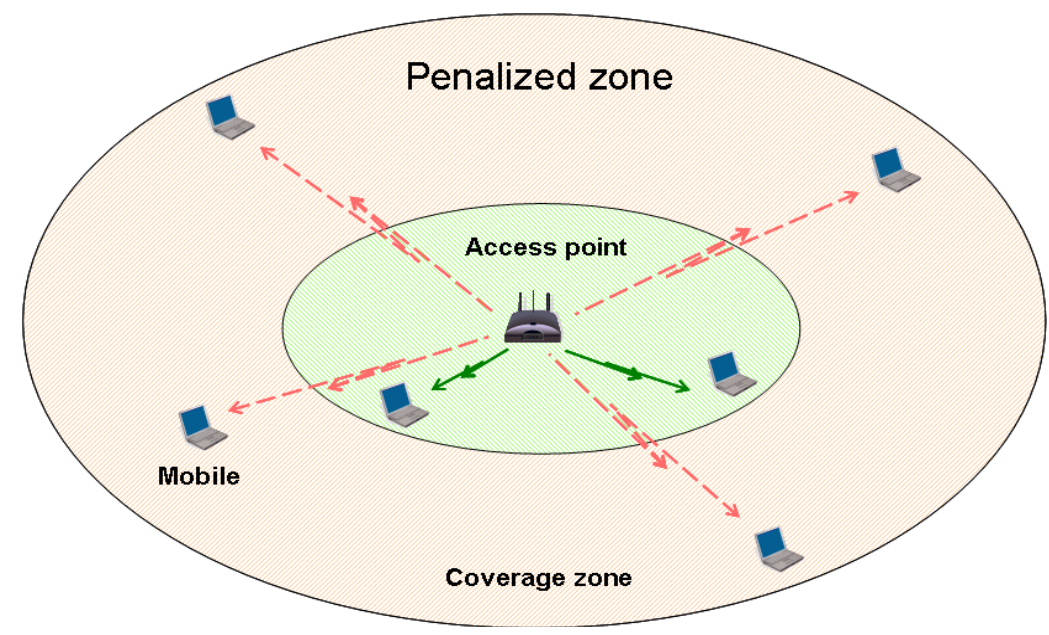

Figure 1. Illustration of Fairness deficiency

In the above equation (2), $t_{c}$ represents a time constant for the moving average. From the above equation, it could sum up that PF scheduling affects the relative preferences with the good channels rather than absolute preference. In the wireless environment where the fading has the greatest impact on the channel state and fluctuates in a random manner and when the number of users are small and if all the users are in the bad channel state, then the throughput will be very low regardless of whoever is scheduled. On the other hand when the number of users is high, there are very high probability that some users can be in a very good channel state. Therefore, the cell throughout gain can be increased by scheduling them first to utilize the fast-fading characteristics. However, the advantage of the multiuser diversity cannot be realised in round 
International Journal of Wireless \& Mobile Networks (IJWMN) Vol. 7, No. 2, April 2015

robin (RR) scheduling. In RR scheduling, the scheduling order is determined well in advance. In this scheduling, each user is given equal amount of time. Based on the channel state, the transmitting rate can be adjusted in RR but the order of service cannot be adjusted. The other schedulers such as IWFQ, C-IFQ, SBFA and WFS select the users based on the channel condition although they have very less information on the channel state. These scheduler delays the service to the users in bad channel state temporarily and schedules the lagging users. As a result, the users can experience frequent packet drops and the lesser throughput when compared with the opportunistic scheduler like PF.

In majority of the real time conditions PF is implemented because of the simplicity. PF scheduler requires per flow queuing which is common to the scheduling algorithms. In core networks, there would be huge amount of per flow queues which creates a huge burden in the core network. Apart from this, PF also requires the channel state information from each user. To do so, BS transmits the broadcast pilot symbol periodically, and the collects the channel state information from each user regularly. Moreover, additional control channel is required to provide the feedback on the channel state, but this depends on the number of users. As the users increases the cost of implementing it also increases.

\section{Cell throughput of the PF scheduler algorithm}

The cell throughput of the PF scheduler algorithm is calculated as indicated in [11], and the associated mathematical considerations are developed based on that paper. The average rate for the user $k\left(\widetilde{R_{k}}[n]\right)$ gets stable as the time goes by because the feasible rate process is stationary. Therefore, the user $k$ 's long term average can be written as

$$
\begin{gathered}
T_{k}=\lim _{n \rightarrow \infty} \widetilde{R_{k}}[n] \\
=\lim _{n \rightarrow \infty} E\left\{R_{k}[n] I_{k}[n]\right\} \\
=\lim _{n \rightarrow \infty} E\left\{R_{k} I_{k}=1\right\} . E\left\{I_{k}\right\} \text { is the expected feasible rate. }
\end{gathered}
$$

The preference metric for user $k$ can be written as $\Gamma_{k}[n]=Z_{k}[n] / \widetilde{Z_{k}}[n]$ at slot $n$. Since $Z_{k}[n]$ does not depend on the time and by omitting the time index the previous equation can be written as:

$$
\Gamma_{k}[n]=Z_{k} / \widetilde{Z_{k}}[n]
$$

The maximum preference for all the users except $k$ can be denoted as $\Gamma_{k-}$, where $\Gamma_{k}>\Gamma_{k-}$ and hence its probability can be written as $\operatorname{Pr}\left\{\Gamma_{k}>\Gamma_{k-}\right\}$. The average throughput for a user for long term can be written as:

$$
\begin{aligned}
T_{k}=\operatorname{Pr} & \left\{\Gamma_{k}>\Gamma_{k-}\right\} \cdot E\left\{R_{k} / \Gamma_{k}>\Gamma_{k-}\right\} \\
& =\int_{0}^{\tilde{R}} r \frac{d}{d r} \operatorname{Pr}\left\{R_{k} \leq r \text { and } \Gamma_{k}>\Gamma_{k-}\right\} d r
\end{aligned}
$$

In the above equation $\tilde{R}$ indicates the maximum feasible rate. 
International Journal of Wireless \& Mobile Networks (IJWMN) Vol. 7, No. 2, April 2015

$$
=\int_{0}^{\tilde{R}} r \frac{d}{d r} \operatorname{Pr}\left\{\Gamma_{k} \leq \varsigma(r) \text { and } \Gamma_{k}>\Gamma_{k-}\right\} d r
$$

After manipulation we obtain the following probability density function (PDF) for $\Gamma_{k}$ denoted by $f \Gamma_{k}(t)$.

$$
T_{k}=\int_{\varsigma(0)}^{\zeta(\infty)} \xi(t) f \Gamma_{k}(t) F_{k-}(t) d t
$$

In our simulation the user is assumed to be static/stationary at one location. Hence in the mathematical analysis produced above the multi user diversity is not taken into considerations. The cell throughput that is derived above is based on the assumption that the user is static/stationary and the shadowing effect is given. In the linear model, the feasibility rate is linearly proportional to the SNR. So this means that the linear model is not accurate. Since the SNR is linearly proportional to feasibility rate, this model can produce some un-reasonable high data rates (HDRs) and gives rise to infinite throughput. On the other hand, in logarithmic model, link adaptation is performed by the adaptive modulation schemes. Among the adaptive modulation schemes, the M-level Quadrature amplitude (M-QAM) shows a good potential and the value of $\mathrm{M}$ is set to two. Therefore, in a good channel condition the large $\mathrm{M}$ is used and in a bad channel condition the small $\mathrm{M}$ is used. Hence, the transmission rate is related to SNR in logarithmic manner as indicated in [14][15][16].

\section{Test setup and results}

The benchmarking tests were performed and compared with the simulation results in [11], in this testing more practical approach were taken. The APN Aggregate Maximum Bit Rate (APNAMBR) applicable for EPS access is a Quality of Service (QoS) policing parameter that allows the network to control the maximum rate of payload traffic received or transmitted on a PDN connection. The Maximum Bit Rate (MBR) is the corresponding parameter applicable for GPRS access. The CPG supports an APN-AMBR and MBR value between 4 kilobits per second (kbps) and 256 megabits per second (Mbps) in both the uplink and downlink direction. Hence, max downlink speed will be $256 \mathrm{Mbps}$ and not $300 \mathrm{Mbps}$.For attaining this, there is a need to either disable the policing or use two APNs of each $256 \mathrm{Mbps}$ max. Gold will get $50 \%$ of the speed offered by the network as max based on CAT3 Speed (100 Mbps in DL and 50 Mbps in UL). Silver will get $25 \%$ of the max speed and Bronze will get around $12 \%$ of the Max speed. This is based on the subscription rent the subscribers pay to the operator for the service. But apart from the subscription plans, the location of the subscriber and the radio channel also plays an equally important role in deciding the throughput for the subscribers. For example, assuming that the Gold subscriber travels to the cell edge, just because he has subscribed for the Gold service It is not possible to negate the impact of the channel condition in the throughput and hence a trade-off between the channel condition and subscription plan has to be made. The proportional fair is based on the trade off between channel quality and average rate per user. In normal scenario, the users at good channel condition and with higher CQI will be allocated good amount of capacity while the users at the cell edge and with poor channel condition will be allocated fewer or no resources in-order to maintain the system performance. However, in PF algorithm, a trade off between system performance and user fairness is delivered to keep the customers in the cell edge or in the bad radio environment satisfied. The following scenarios were tested by means of a script in the test environment: 
International Journal of Wireless \& Mobile Networks (IJWMN) Vol. 7, No. 2, April 2015

Scenario 1: Gold, Silver and Bronze UEs are subjected to test by means of the FTP test

Scenario 2 : Gold and Bronze

Scenario 3 :Gold and Silver

Scenario 4 :Silver and Bronze

Scenario 5 : Gold only

Scenario 6 : Silver Only

Scenario 7 : Bronze only

Table 1 explains the various testing scenarios. Each scenarios are tested independently and also with various combinations. Table 2 and Table 3 provides the details about the radio access network (RAN) configurations made in the network exclusively for the benchmarking testing. Table 4 to Table 7 provides the benchmarking results in the tabular format. Figure 2 provides benchmarking results in the graphical form. The test results show that, when a Gold, Silver and Bronze users are tested individually in the good channel conditions, the throughput achieved is almost the same. Unlike, in the bad channel conditions different priority is set for different subscription types. The Gold and Silver are set as medium priority, but the Bronze users are set as high priority. Apart from the subscription type, the channel condition also plays a major part. Since the Bronze users are with the minimum subscription, the channel condition will further bring down the throughput rate. So that is why the Bronze users are set with high priority in bad channel condition. TEMS Investigation, Sony Xperia smart phones capable of LTE, scripts for testing various subscriptions and SIM Card configured with different ARP with Gold, Silver and Bronze profiles in the HSS.

Table 1. Fairness testing scenarios

\begin{tabular}{|c|c|}
\hline No & Scenarios \\
\hline 1 & Case1-Gold User \\
\hline 2 & Case2-Gold User + Silver User \\
\hline 3 & Case3-Gold User + Silver User +Bronze \\
\hline 4 & Case4-Gold User + Bronze User \\
\hline 5 & Case5-Silver User + Bronze User \\
\hline 6 & Case6-Silver User \\
\hline 7 & Case7-Bronze User \\
\hline
\end{tabular}


International Journal of Wireless \& Mobile Networks (IJWMN) Vol. 7, No. 2, April 2015

Table 2. RAN configuration for the testing

\begin{tabular}{|c|c|}
\hline Parameter Default Value & Value \\
\hline Downlink Aggregate MBR for the UE in the MME & $\begin{array}{l}10000000 \\
\mathrm{kbps}\end{array}$ \\
\hline Uplink Aggregate MBR for the UE in the MME & $\begin{array}{l}10000000 \\
\mathrm{kbps}\end{array}$ \\
\hline Downlink Aggregate MBR of an APN in the MME & $\begin{array}{l}10000000 \\
\mathrm{kbps}\end{array}$ \\
\hline Uplink Aggregate MBR of an APN in the MME & $\begin{array}{l}10000000 \\
\mathrm{kbps}\end{array}$ \\
\hline $\begin{array}{l}\text { Highest allowed ARP priority level for a non-GBR bearer in the } \\
\text { MME }\end{array}$ & 1 \\
\hline Allowed value of PCI for a non-GBR bearer in the MME & yes \\
\hline Allowed value of PVI for a non-GBR bearer in the MME & no \\
\hline MBR Downlink & $256000 \mathrm{kbps}$ \\
\hline MBR Uplink & $256000 \mathrm{kbps}$ \\
\hline GBR Downlink & $256000 \mathrm{kbps}$ \\
\hline GBR Uplink & $256000 \mathrm{kbps}$ \\
\hline Highest allowed ARP priority level for a GBR bearer in the MME & 1 \\
\hline Allowed value of PCI for a GBR bearer in the MME & yes \\
\hline Allowed value of PVI for a GBR bearer in the MME & no \\
\hline
\end{tabular}

Table 3. Network configuration for testing fairness

\begin{tabular}{|c|c|c|c|}
\hline QCI & Parameter & Value & Remarks \\
QCI6 & $\begin{array}{c}\text { Scheduling } \\
\text { algorithm }\end{array}$ & 3 & PFS_MEDIUM (GOLD) \\
\hline QCI8 & $\begin{array}{c}\text { Scheduling } \\
\text { algorithm }\end{array}$ & 3 & PFS_MEDIUM (Silver) \\
\hline QCI9 & $\begin{array}{c}\text { Scheduling } \\
\text { algorithm }\end{array}$ & 2 & PFS_HIGH (Bronze) \\
\hline QCI6 & DL Min bit rate & 40000 & Kbps \\
\hline QCI8 & DL Min bit rate & 20000 & Kbps \\
\hline QCI9 & DL Min bit rate & 10000 & Kbps \\
\hline QCI6 & Relative priority & 57 & $\begin{array}{c}\text { Relative priority keeping 100\% as the } \\
\text { max }\end{array}$ \\
\hline QCI8 & Relative priority & 28 & $\begin{array}{c}\text { Relative priority keeping 100\% as the } \\
\text { max }\end{array}$ \\
\hline QCI9 & Relative priority & 15 & $\begin{array}{c}\text { Relative priority keeping 100\% as the } \\
\text { max }\end{array}$ \\
\hline
\end{tabular}


International Journal of Wireless \& Mobile Networks (IJWMN) Vol. 7, No. 2, April 2015

Table 4. LTE QOS Benchmark Result - Averages Values

\begin{tabular}{|l|c|c|c|}
\hline \multirow{2}{*}{ Scenarios } & \multicolumn{2}{c|}{ LTE QOS Benchmark Result } \\
\cline { 2 - 4 } & $\begin{array}{c}\text { Gold } \\
\text { User }\end{array}$ & $\begin{array}{c}\text { Silver } \\
\text { User }\end{array}$ & $\begin{array}{c}\text { Bronze } \\
\text { User }\end{array}$ \\
\cline { 2 - 4 } Case1-Gold User & 27.75 & - & - \\
\hline Case2-Gold User + Silver User & 23.55 & 16.59 & - \\
\hline Case3-Gold User + Silver User +Bronze & 24.48 & 14.96 & 7.76 \\
\hline Case4-Gold User + Bronze User & 23.47 & - & 10.57 \\
\hline Case5-Silver User + Bronze User & - & 24.53 & 18.16 \\
\hline Case6-Silver User & - & 30.62 & - \\
\hline Case7-Bronze User & - & - & 26.78 \\
\hline
\end{tabular}

Table 5. QoS for Gold subscription

\begin{tabular}{|l|c|c|c|c|c|}
\hline \multirow{2}{*}{ Scenarios } & \multicolumn{5}{c|}{ Gold } \\
\cline { 2 - 6 } & Test 1 & Test 2 & Test 3 & Test 4 & Test 5 \\
\hline Case1-Gold User & 27.3 & 31.59 & 29.08 & 26.26 & 24.52 \\
\hline Case2-Gold User + Silver User & 20.36 & 22.82 & 26.78 & 26.03 & 21.79 \\
\hline Case3-Gold User + Silver User +Bronze & 21.42 & 26.87 & 25.28 & 24.99 & 23.87 \\
\hline Case4-Gold User + Bronze User & 28.89 & 21.47 & 26.04 & 17.39 & 23.59 \\
\hline Case5-Silver User + Bronze User & - & - & - & - & - \\
\hline Case6-Silver User & - & - & - & - & - \\
\hline Case7-Bronze User & - & - & - & - & - \\
\hline
\end{tabular}

Table 6. QoS for Silver subscription

\begin{tabular}{|l|c|c|c|c|c|}
\hline \multirow{2}{*}{ Scenarios } & \multicolumn{5}{c|}{ Silver } \\
\cline { 2 - 6 } & Test 1 & Test 2 & Test 3 & Test 4 & Test 5 \\
\hline Case1-Gold User & - & - & - & - & - \\
\hline Case2-Gold User + Silver User & 19.05 & 17.03 & 10.19 & 16.06 & 20.66 \\
\hline Case3-Gold User + Silver User +Bronze & 16.11 & 16.27 & 14.24 & 14.05 & 14.14 \\
\hline Case4-Gold User + Bronze User & - & - & - & - & - \\
\hline Case5-Silver User + Bronze User & 26.51 & 24.95 & 22.8 & 22.32 & 26.08 \\
\hline Case6-Silver User & 28.39 & 31.67 & 30.66 & 31.37 & 31.05 \\
\hline Case7-Bronze User & - & - & - & - & - \\
\hline
\end{tabular}


International Journal of Wireless \& Mobile Networks (IJWMN) Vol. 7, No. 2, April 2015

Table 7. QoS for Bronze subscription

\begin{tabular}{|l|c|c|c|c|c|}
\hline \multirow{2}{*}{\multicolumn{1}{|c|}{ Scenarios }} & \multicolumn{5}{c|}{ Bronze } \\
\cline { 2 - 6 } & Test 1 & Test 2 & Test 3 & Test 4 & Test 5 \\
\hline Case1-Gold User & - & - & - & - & - \\
\hline Case2-Gold User + Silver User & - & - & - & - & - \\
\hline Case3-Gold User + Silver User +Bronze & 8.27 & 7.67 & 7.32 & 8.07 & 7.51 \\
\hline Case4-Gold User + Bronze User & 6.17 & 7.71 & 6.53 & 13.34 & 19.14 \\
\hline Case5-Silver User + Bronze User & 17.71 & 18.41 & 18.45 & 20.7 & 15.55 \\
\hline Case6-Silver User & - & - & - & - & - \\
\hline Case7-Bronze User & 26.72 & 30.7 & 15.32 & 30.17 & 31 \\
\hline
\end{tabular}

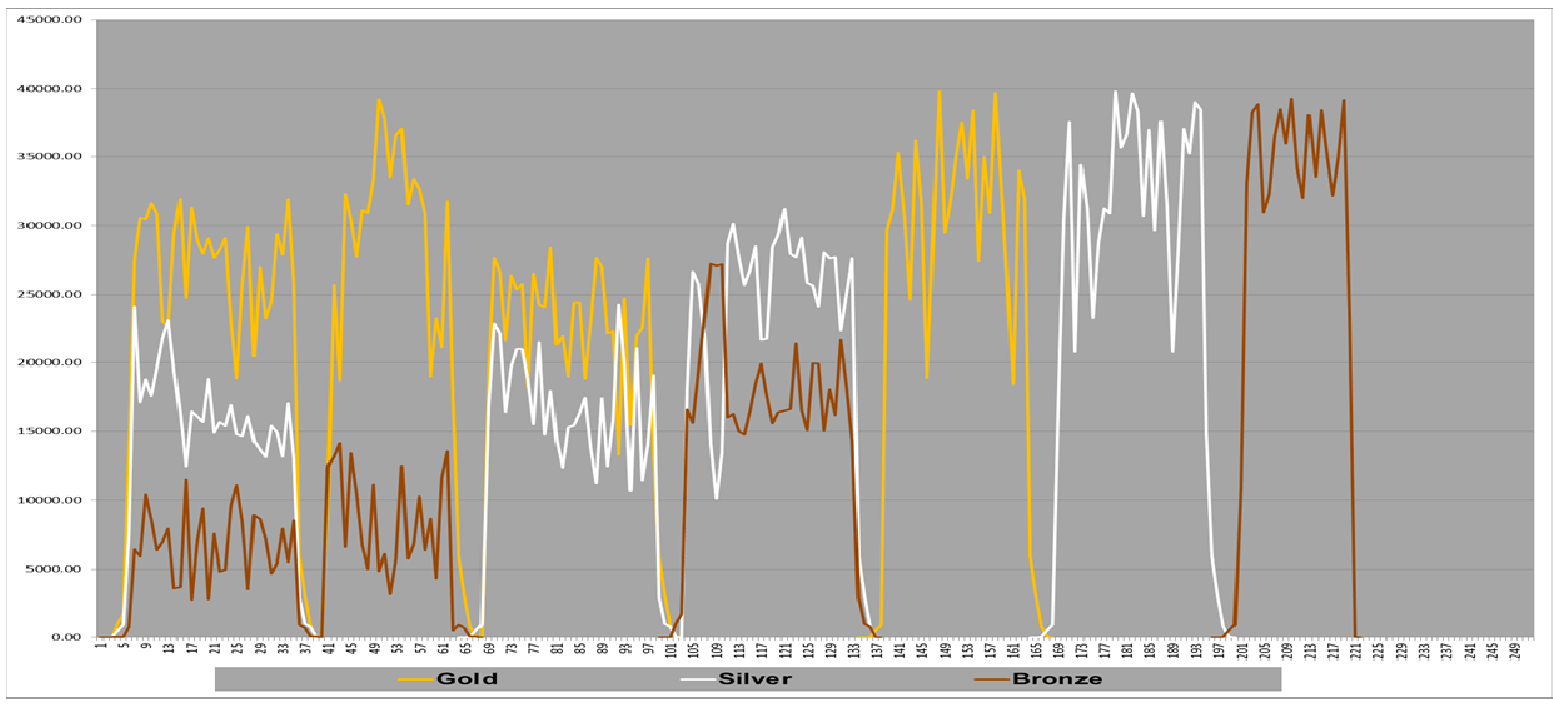

Figure 2. Benchmarking test results for different scenarios (Subscription plan Vs Throughput)

The benchmarking environment of the cell radius considered is around $1 \mathrm{Km}$. The downlink channel slot length is $1.67 \mathrm{~ms}$. The BS transmission power is fixed at $10 \mathrm{~W}$. The standard deviation of the shadow fading is around $8 \mathrm{~dB}$ and path loss exponent is around 4db. In [11], the simulation results are derived for the cell throughput of the PF scheduler in the single cell environment. The simulation results are not reproduced again in this paper. As per the simulation results, when the number of subscribers increases the overall system throughput increases in the beginning, and then it stabilizes around $3 \mathrm{bps} / \mathrm{Hz}$ without further increase. The same behaviour can be observed in our benchmarking tests. In Figure 2, the Gold, Silver and Bronze subscribers are tested in a good channel condition individually using separate scripts. In the benchmarking result, the number of subscribers is not shown explicitly as in simulation. Since it is carried out in a live network site, it is assumed that there will be lot of subscribers trying to access the data from the network. This assumption satisfies the condition based on which the simulation in [11] was carried out. The standard deviation and the median SNR show the increased throughput as a witness in the benchmarking tests. The various scenarios reported in the graph in Figure 2 show 
International Journal of Wireless \& Mobile Networks (IJWMN) Vol. 7, No. 2, April 2015

that the different subscription in the bad channel condition with various priorities like high and medium that enables the subscribers from achieving different levels of throughput as per the subscriptions. So on the whole, the benchmarking test results confirm the result obtained in [11] by simulations. The PF scheduler is compared with other competing schedulers proposed in [5][6] to maximize the average throughput by satisfying the QoS constrains. In [6], each users requires a certain amount of time slots reserved to be served and in [5] the normalized throughput of the user is equalized and priori weight is allocated based on the users channel condition and QoS requirement.

\section{CONCLUSION}

In traditional wireless networks, fairness was not considered because the cellular channels are assigned based on pre-reservation strategies, whereas in modern wireless technologies like in 4G technology, the reservation based allocation will not serve the purpose of the subscribers and the network operators. These new technologies seriously consider fairness and user throughput as one of the major requirement. Hence, several scheduling algorithms have been put forth by many researchers to achieve this feat. Among them, PF scheduling algorithm is a very popular one, since it offers various degree of flexibility for the operators to serve the people based on the location, channel environment, service subscription so on so forth. Among this, channel condition plays a vital role in user throughput and PF offers this required balancing.

The PF scheduler is analysed, and then the mathematical expression for the cell throughput has been re-produced for the readers convenience based on [11]. In this analysis, the authors have adopted a slightly modified metric. This approach supports the relationship between the transmission rate and SNR. The authors have also verified the accuracy of the mathematical expressions derived with the simulation results. In this paper, more practical approach has been taken and performed the benchmarking tests and analysed the performance of the PF. The users were classified as Gold, Silver and Bronze based on the service subscription. Combination of various scenarios has been tested as specified in Table 1. The outcomes of the benchmarking tests were compared with [11] and the throughput levels obtained for various scenarios are found almost the same. Since the benchmarking tests in this paper was carried in a live network, the factors affecting the throughput were not considered separately as in [11], it is assumed that all the factors affecting the throughput will be at play in practical environment.

\section{Reference:}

[1]. F. Kelly, "Charging and rate control for elastic traffic," Eur. Trans.Telecommun., vol. 8, no. 1, pp. 33-37, Jan. 1997. S. Liu and V. Bharghavan, "Fair scheduling in wireless packet networks, IEEE/ACM Trans. Netw., vol. 7, no. 4, pp. 473-489, Aug. 1999.

[2]. A. K. Parekh and R. G. Gallager, "A generalized processor sharing approach to flow control in integrated services networks: The single-node case, IEEE/ACM Trans. Netw., vol. 1, no. 3, pp. 344357, Jun. 1993.

[3]. T. S. E. Ng, I. Stoica, and H. Zhang, "Packet fair queuing algorithms for wireless networks with location-dependent errors," in Proc. IEEE INFOCOM, San Francisco, CA, 1998, pp. 1103-1111.

[4]. A. Jalali, R. Padovani, and R. Pankaj, "Data throughput of CDMA-HDR a high efficiency-high data rate personal communication wireless system, in Proc. IEEE VTC, Tokyo, Japan, 2000, pp. 18541858 .

[5]. S. Borst and P. Whiting, "Dynamic rate control algorithms for HDR throughput optimization," in Proc. IEEE INFOCOM, Anchorage, AK, 2001, pp. 976-985. 
International Journal of Wireless \& Mobile Networks (IJWMN) Vol. 7, No. 2, April 2015

[6]. X. Liu, E. K. P. Chong, and N. B. Shroff, "Opportunistic transmission scheduling with resourcesharing constraints in wireless networks," IEEE J. Sel. Areas Commun., vol. 19, no. 10, pp. 20532064, Oct. 2001.

[7]. "A framework for opportunistic scheduling in wireless networks, Comput. Netw., vol. 41, no. 4, pp. 451-474, Mar. 2003.

[8]. Y. Liu and E. Knightly, "Opportunistic fair scheduling over multiple wireless channels, in Proc. IEEE INFOCOM, San Francisco, CA, 2003, pp. 1106-1115.

[9]. X. Qin and R. Berry, "Exploiting multiuser diversity for medium access control in wireless networks," in Proc. IEEE INFOCOM, San Francisco, CA, 2003, pp. 1084-1094.

[10]. P. Viswanath, D. N. C. Tse, and R. Laroia, "Opportunistic beamforming using dumb antennas," IEEE Trans. Inf. Theory, vol. 48, no. 6, pp. 1277-1294, Jun. 2002.

[11]. Jin-Ghoo Choi and Saewoon Bhak, "Cell-Throughput Analysis of the Proportional Fair Scheduler in the Single-Cell Environments", IEEE Transaction on Vehicular Technology.,vol.56,no.2, pp. 766778, March.2007.

[12]. L. Massoulié and J. Roberts, "Bandwidth sharing: Objectives and algorithms", IEEE/ACM Trans. Netw., vol. 10, no. 3, pp. 320-328, Jun. 2002.

[13]. J. M. Holtzman, "Asymptotic analysis of proportional fair algorithm," in Proc. IEEE PIMRC, San Diego, CA, 2001, pp. 33-37.

[14]. H. J. Kushner and P. A. Whiting, "Convergence of proportional-fair sharing algorithms under general conditions," IEEE Trans.Wireless Commun., vol. 3, no. 4, pp. 1250-1259, Jul. 2004.

[15]. S. Catreux, P. F. Driessen, and L. J. Greenstein, "Data throughputs using multiple-input multipleoutput (MIMO) techniques in a noiselimited cellular environment," IEEE Trans. Wireless Commun., vol. 1, no. 2, pp. 226-234, Apr. 2002.

[16]. A. J. Goldsmith and S.-G. Chua, "Variable-rate variable-power MQAM for fading channels," IEEE Trans. Commun., vol. 45, no. 10, pp. 1218-1230, Oct. 1997.

\section{Author Biographies:}

Ramprasad Subramanian is an experienced telecom engineer in the field of $2 \mathrm{G} / 3 \mathrm{G}$ and LTE/LTE-A. He holds M.S (By research) in Information and Communication from Institute of Remote Sensing, Anna University (India)(2007) and Bachelors of Engineering in Electronics and Communication engineering from Bharathidasan University (2001)(India). He has done many projects in the area of 2G/3G and LTE. He has done many consultative projects across Africas/Americas/Asia etc. He was the recipient of India's best invention award for the year 2004 from Indian Institute of Management Ahmadabad and Government of India. His current research focuses on $4 \mathrm{G}$ mobile networks and vehicular Ad hoc networks.

Roshanak Heidari is PHD student at UTS in Centre for Real-Time Information Networks (CRIN). She holds a Master's of Science in Electrical Engineering from Islamic Azad University, Science and Research Branch(Iran)(2007), and a Bachelor of Science Degree in Electrical Engineering ,from Tabriz University (2004). Her current research work focuses on Optimization for Resource Allocation with Constraints in Wireless Networks.

Dr Kumbesan Sandrasegaran is an Associate Professor at UTS and Centre for Real-Time Information Networks (CRIN). He holds a PhD in Electrical Engineering from McGill University (Canada)(1994), a Master of Science Degree in Telecommunication Engineering from Essex University (1988) and a Bachelor of Science (Honours) Degree in Electrical Engineering (First Class) (1985). His current research work focuses on two main areas (a) radio resource management in mobile networks, (b) engineering of remote monitoring systems for novel applications with industry through the use of embedded systems, sensors and communications systems. He has published over 100 refereed publications and 20 consultancy reports spanning telecommunication and computing systems.

AMA Dhanraj brings in expertise in telecom domain over 13 years in various cross domains across telecom and different access technologies and holds a Bachelor of Engineering in Electronics and 
International Journal of Wireless \& Mobile Networks (IJWMN) Vol. 7, No. 2, April 2015

communication from the University of Madras - India (2001), has contributed to the success of projects in GSM,WCDMA and LTE as a consultant in Asia/Africa/Middle east, he is currently specializing in LTE/LTE-A and focus on solution development based on RAN products.

Karthik $\mathbf{S}$ is engineer turned Project Manager bringing in rich experience of his technical expertise into Project Management, Holds a Bachelor of Engineering in Electronics and communication from the Bharathidasan University- India(2000) and MBA in Information technology from Symbiosis(Dubai), has delivered over past 14 years various support related activities in Asia-Pacific/Africa/Europe and PMP certified which he uses his skills to the fullest for effective Project Management contributing to the bottom line of the organization, Pursuing PGMP currently. 